\title{
Crystal engineering of organic compounds for solid-state reactions
}

\author{
Demetrius C. Levendis ${ }^{1}$, Sanaz Khorasani ${ }^{1}$, Delbert S. ${ }^{1}$, Manuel A. Fernandes ${ }^{1}$ \\ ${ }^{1}$ Molecular Sciences Institute, University Of The Witwatersrand, Johannesburg, South Africa \\ E-mail: demetrius.levendis@wits.ac.za
}

The topochemical criteria first outlined by Schmidt and coworkers has provided a useful guideline as to whether or not a reaction will occur in the solid state. Potentially reacting molecules should typically be less than $4.2 \AA$ apart and suitably oriented for dimerization. In trying to design crystals in which molecules are suitably aligned to react, one often looks at suitable synthons that can assemble according to hydrogen bonding, electrostatic interactions, weaker $\mathrm{C}-\mathrm{H} \cdots \mathrm{O}$ type interactions and finally dispersion. In our recent work on the [2+2] photodimerization of o-ethoxy-cinnamic acid (OETCA) [1] we have shown some exceptions to the topochemical rule in the case of the a-polymorph photo-irradiated at two different temperatures. Hydrogen bonded $\mathrm{C}=\mathrm{O} \cdots \mathrm{H}-\mathrm{O}-\mathrm{C}$ pairs of these molecules form sheets of these pairs held together by $\mathrm{C}-\mathrm{H} \cdots \mathrm{O}$ interactions. Finally, the stacking of these sheets is determined largely by dispersion forces, which in the case of OETCA results in three different polymorphs, each of which reacts differently in the solid state.

In reactions involving a thermal [4+2] Diels-Alder dimerization, electron donor/acceptor (EDA) interactions (which are better described as dispersion interactions) influence the way in which pairs of dithiin (acceptor) and anthracene (donor) molecules assemble, forming heteromolecular crystals of charge-transfer (CT) complexes that can potentially react in the solid state. In these CT complexes it is therefore desirable to not only form a CT pair, but for this pair to crystallize in stacks of alternating donor and acceptor molecules that conform to the topochemical criteria. Typically the crystal structures consist of stacks of alternating electron donor and acceptor molecules in a 1:1 ratio. The crystals can then undergo a thermally induced solidstate (SS) Diels - Alder reactions in a single-crystal-to-single-crystal (SCSC) fashion, with the dithiin molecules as the dienophiles and anthracenes as the diene. The nature of the R-groups on the dithiin (cycloalkanes, aromatic groups or chiral molecules) and X-groups on the anthracene $(\mathrm{H}, \mathrm{Me}, \mathrm{Br}$, allyl) critically affect the packing of the molecules and hence their reactivity. Reactions can take place at temperatures well below their melting points depending on the constraints imposed by the reaction cavity $[2,3]$.

In this paper we discuss both the photodimerization of OETCA and the effect of the dithiin substituents, R1 and R2, and of the anthracene substituents, $X$, on the formation of CT complexes and on their reactivity.

[1] Fernandes M. A.and Levendis D.C. (2016). CrystEngComm. 18, 7363-7376.

[2] Khorasani S. and Fernandes M.A. (2013). Cryst. Growth Des. 13, 5499-5505.

[3] Khorasani S., Botes D. S., Fernandes M. A. and Levendis D. C. (2015). CrystEngComm. 17, 8933-8945.
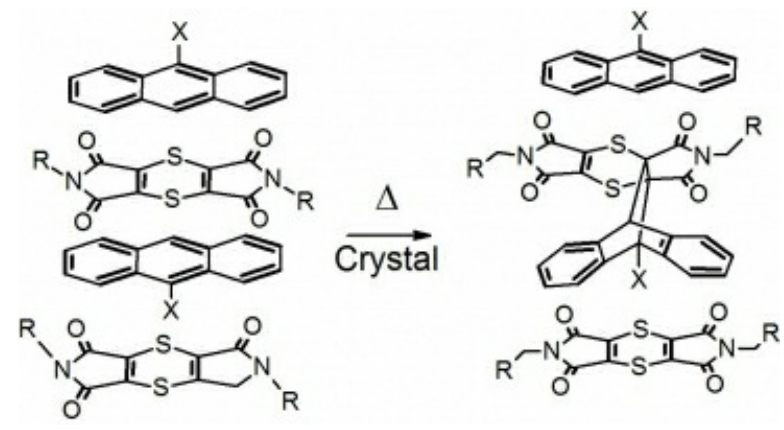

Keywords: Solid-state reaction, Diels-Alder, photodimerization 